\title{
A PHOTOGRAPHIC STUDY OF ELECTROLYTIC CELLS.
}

By Rolla R. Ramsey.

$\mathrm{W}$

HILE making preliminary experiments with a view of using Töpler's "Schlierenapparat" 1 as a possible means for the study of vibrating films as suggested by Dr. A. G. Webster, the idea occurred to me of using the apparatus to study electrolytic action. The apparatus, as shown in diagram, is arranged as follows: $S_{1}$ is an illuminated adjustable slit, the image of which, by means of the lens, $L_{1}$, is thrown on a second adjustable slit, $S_{2}$. An electrolytic

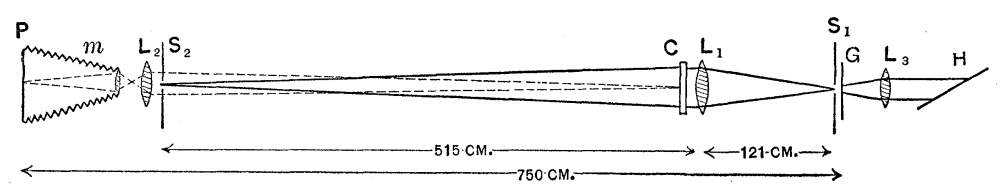

Fig. 1.

cell, $C$, is placed between $L_{1}$ and $S_{2}$ near to $L_{1}$. The image of $C$ is projected on the plate in the camera at $P$ by means of two lenses $L_{2}$ and $M . \quad L_{1}$ is an achromatic lens of $5 \mathrm{~cm}$. diameter and $93 \mathrm{~cm}$. focal length. $L_{2}$ is a photographic lens of $25.5 \mathrm{~cm}$. focal length. $M$ is a $3 \mathrm{~cm}$. focal length microscope objective. $S_{1}$ is illuminated with sunlight by means of a cylindrical lens $L_{3}$ and a heliostat $H$.

The principle of the "Schlieren-methode" is, that if the medium between $S_{1}$ and $S_{2}$ is homogeneous, then the image of $S_{1}$ will be brought to a sharp focus at $S_{2}$, which has been placed at the conjugate focus of $S_{1}$. If the slit of $S_{2}$ is made to coincide with the image of $S_{1}$, then the illumination on the screen $P$ will be uniform. Moving the slit $S_{2}$ up will intercept the light and the screen will suddenly become dark. If the medium between $S_{1}$ and $S_{2}$ is not homogeneous, then the image of $S_{1}$ will not be sharp and moving $S_{2}$ up will intercept the light from certain portions of the field, causing it to be blotched or spotted, "Schlieren." Intercepting the lower part of the image will cause certain portions to be light, while intercepting the upper will illuminate what was dark in the first case and darken the former bright portions. The homogeneity of

1 Pogg. Ann., r31, 33 and r8o, r867. 
medium will be violated by heating the air, by inserting a cell in which liquids of different densities are placed, or by an electrolytic cell having a current flowing through it. The cell $C$ is made of two pieces of plane optical glass, the inside dimensions of which are, width $2.2 \mathrm{~cm}$., thickness $.4 \mathrm{~cm}$. The electrodes are made of strips of commercial sheet zinc. The electrolyte is a $10 \%$ solution of sinc sulphate (10 gm. $\mathrm{ZnSO}_{4}$ in $90 \mathrm{cu}$. cm. of water). The solution is a clear liquid, which, when placed in the cell, has every appearance of water to the naked eye.

The accompanying photographs will illustrate the results. No. 2 shows the current passing up through the electrolyte. Since the anode is being dissolved and the cathode plated, we have changes of density at the two electrodes. A dense solution, which is seen to be falling, is formed at the anode. A corresponding light solution, which rises to the surface, is formed at the cathode. The portion between the two electrodes is uniform and, therefore, the appearance is dark. The light in this case is taken from the upper part of the slit. In No. 4 we have the same cell except that the current is reversed, that is, passing down through the solution. The dense solution is falling from the anode and the rare is rising, making a variable density between the two electrodes. Light is taken from the upper part of slit the same as in No. 2. No. I 5 shows vertical electrodes. The current causes a stream of dense solution to flow from the anode. No. I 5 also shows a characteristic stratification of the solution between the two electrodes. A horizontal layer of dense liquid is invariably formed at the lower extremities of the electrodes. Light is taken from the center of the slit. No. I I is taken from a $5 \mathrm{~cm}$. cubical cell made of plate glass with horizontal plate electrodes. The current is passing down through the liquid. The light is taken from the upper part of the slit. Nos. 32 and 33 illustrate the difference in the effect by taking light from the upper or lower portions of the slit. No. 32 was taken from the lower part of the slit, while No. 33 was taken from the upper. They were taken one after the other as quickly as possible, the only change being that of moving $S_{2}$ up a short distance. It will be noticed that one is the exact negative of the other.

The time of exposure varied from one to four seconds, although plates were taken with an ordinary instantaneous shutter.

Clark University, June I4, I899. 
The Physical Review.

No. 2

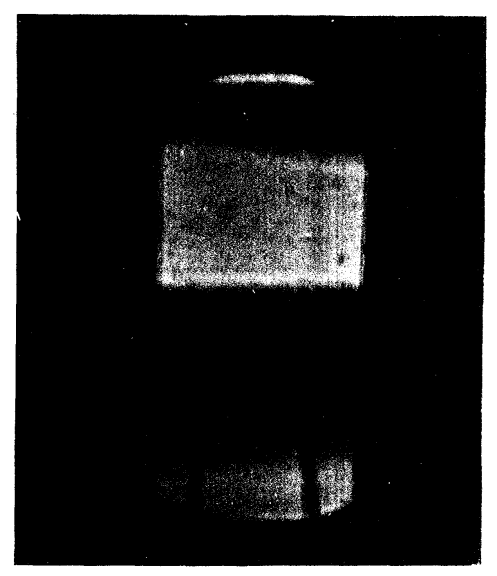

No. 15

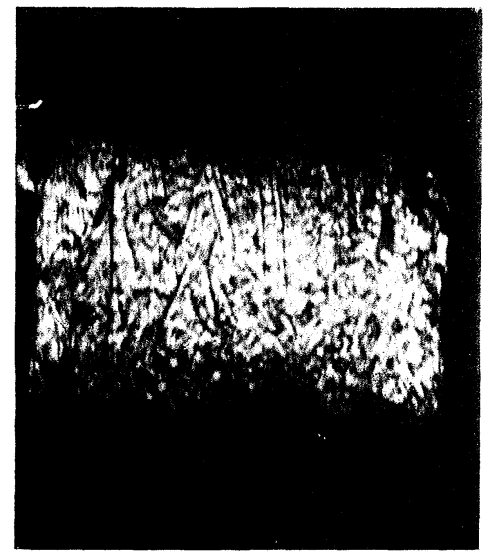

No. 32

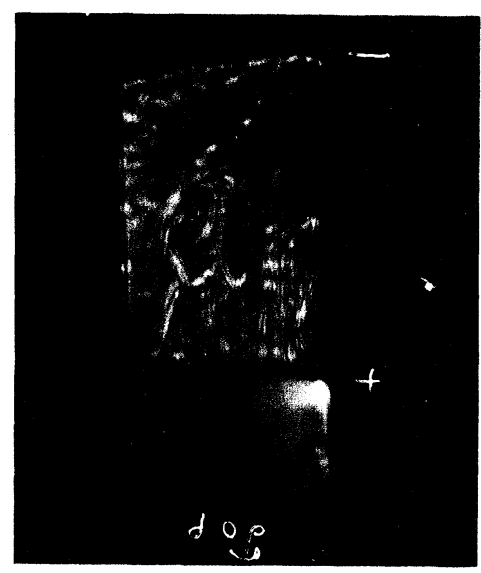

No. 4

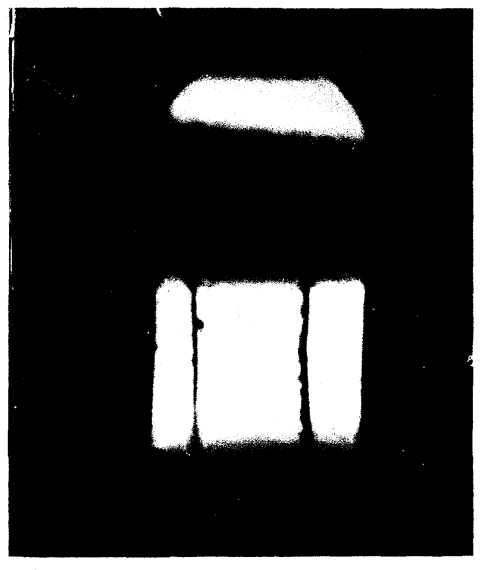

No. I I

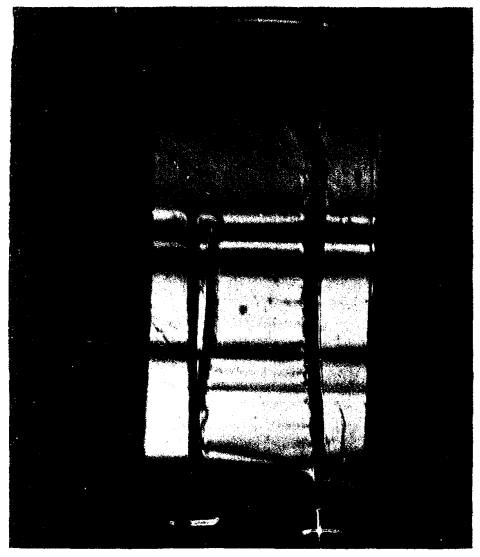

No. 33

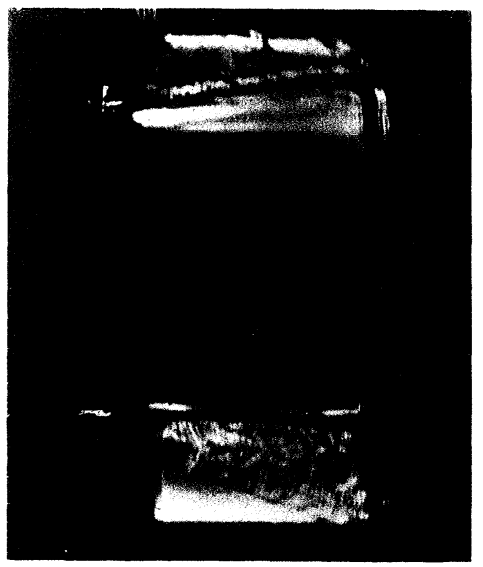

PLATE I. RAMSEY. 
No. 2

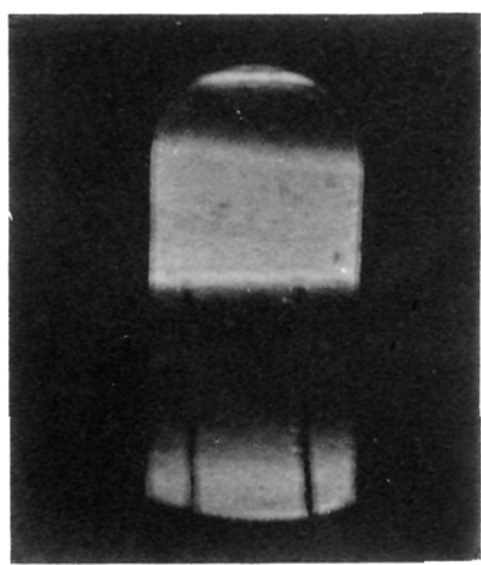

No. 15

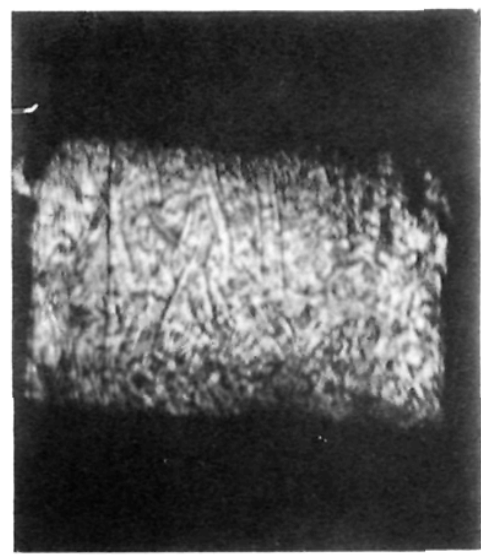

No. $3^{2}$

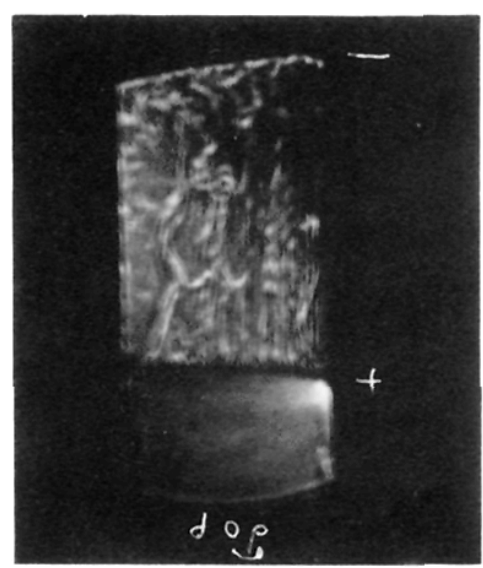

No. 4

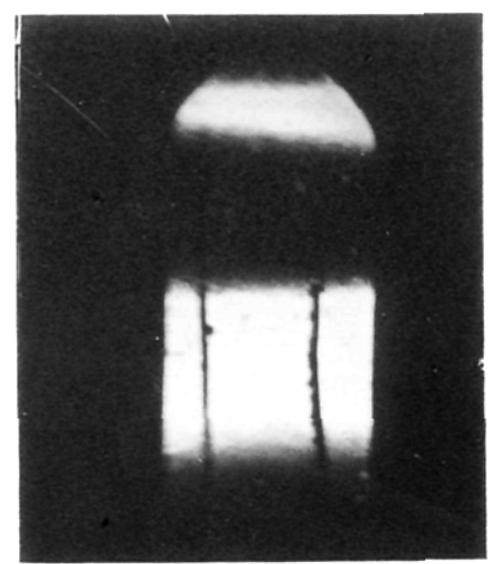

No. I I

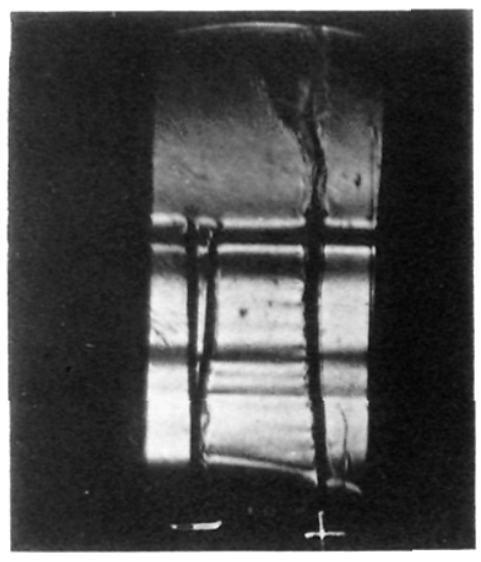

No. 33

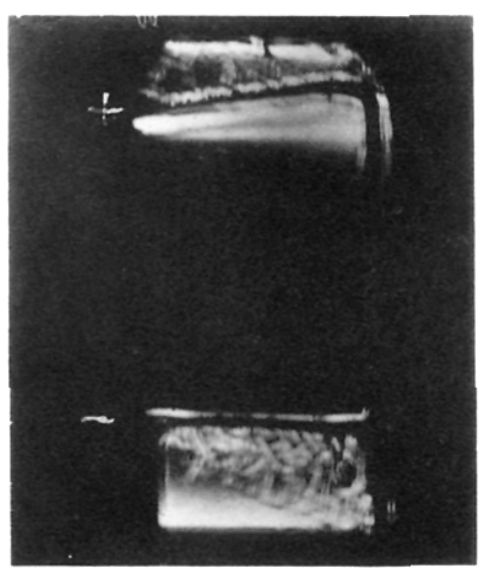

